\title{
Enhancing Shadow Area Using RGB Color Space
}

\author{
M. S. V. Jyothirmai ${ }^{1}$ Dr. K. Srinivas $^{2}$ Dr. V. Srinivasa Rao ${ }^{3}$ \\ Post Graduate Student Professor Professor \\ ${ }^{1,2,3}$ (Department of CSE, VRSEC, Vijayawada, AP, INDIA)
}

\begin{abstract}
Shadow detection and removal from color images is invariably significant for image processing. Existing methods use illuminant invariant methods to detect and minimize shadow which is computationally expensive. RGB color space based shadow removal method is existing one which blurs shadow edges. The performance of this existing method mainly depends on structuring element. To overcome all disadvantages, this paper is aimed to utilize RGB color space with modified structuring element. The effect of shadow is decreased on pixel while increasing values of 3 color channels of pixel using modified approach. Experiment results show that proposed system is computationally inexpensive and can robustly remove shadow from complex images.
\end{abstract}

Keywords: Enhancing Shadow Area, RGB Color space, Shadow Detection, Shadow Removal, Shadow mask,

\section{Introduction}

Image preprocessing involves various steps to enhance image fine features. Some of them include noise type detection, noise removal and Image resolution enhancement. All these are preliminary and accompanied for image. The most unwanted information of image is shadow area. Shadows may lead the misinterpreting of actual objects. Shadow occurs when objects occlude light from light source. Shadows provide rich information about the object shapes as well as light orientations. Sometimes we cannot recognize the original image of a particular object. Shadow in image reduces the reliability of many computer vision algorithms. Shadow often degrades the visual quality of images [1]. Shadow removal in an image is an important pre-processing step for computer vision algorithm and image enhancement. Shadows in images have long been disruptive to computer vision algorithms. They appear as surface features, when in fact they are caused by the interaction between light and objects [2]. This may lead to problems in scene understanding, object segmentation, tracking and recognition. Because of the undesirable effects of shadows on image analysis, much attention was paid to the area of shadow removal over the past decades [3].

Shadows are classified into 2 types including self shadow and cast shadow. Self-shadow is forming when the object objects its self and another is cast shadow forming from light source. Distinction between these types of shadows is important for object recognition like in fig 1. Successful shadow removal aims to remove cast shadows while recognizing self-shadows as part of the object of interest and therefore preserving them [4]. Both cast and self shadow have different brightness value. The brightness of all the shadows in an image depends on the reflectivity of the object upon which they are cast as well as the illumination from secondary light sources. Self-shadow usually have a higher brightness than cast shadows since they receive more secondary lighting from surrounding illuminated objects. One crucial difference between these shadows is their contrast to the background. Usually, self shadows are vague shadows which gradually change intensity and have no clear boundaries. Cast shadows are, on the other hand, hard shadows with sharp shadow boundaries [5].

Existing methods concentrate on finding 1-D illuminant invariant image of given image. Lambertian surfaces assumption is forcing that there is no specular reflectance in image. The Mondrian world and smooth illumination assumptions assume that there is a generally clear signal at each of the boundaries between objects whereas there are no sharp boundaries between shadows and background. Similar work on vague shadow removal can be also found in $[6,7]$ where authors directly utilize a low-pass filter to separate the illumination images.

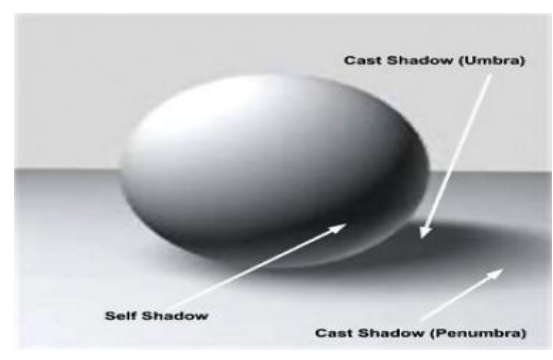

Fig. 1: An Object Showing Cast and Self-Shadows

Recently, Y. Weiss [8] proposed a system to remove shadows from image sequences and Matsushita et al. [9] extended his work. Their methods also based on a decomposition of images into reflectance and illumination. They used an ML estimation to derive the time-invariant reflectance image from the sequences. Since they make a more generic assumption instead of the Mondrian world and smooth illumination, their 
method could remove cast shadows as well as vague shadows. However, the system has limitations for that it could only remove moving shadows in image sequences. All these experiments provide complex solution to a challenging problem [10-13].

\section{Proposed Approach}

Proposed method includes 2 phases having shadow detection phase and shadow removal phase. The goal of the detection phase is to identify the shadowed pixels to be recovered. For shadow detection we present an approach based on statistics of intensity. First, shadow image is considered in RGB color space. Compute size of image to create mask. Mask value will be automatically calculated to grayscale image using graythresh() function.

mask $=1$ - image2binary $($ gray_image, graythresh $($ gray_image $))$

Structuring element (SE) to burr the shadow mask is considered. This consideration is important for individual images as result is varied. We proposed modified structuring element SE $5 \times 5$ array of 0's or 1's. Initially this is $3 \times 3$ array in existing approaches which yields blurred edges and producing more light to shadowed pixels [2]. To overcome the problem we conduct no of experiments on shadow mask values and finally conclude SE as $5 \times 5$ array. In our proposed method we consider

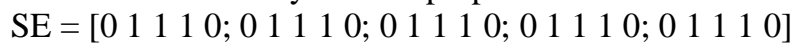

SE value is passed to imerode() function to detect shadow and light areas. Function imerode() erodes the grayscale, binary, or packed binary image, returning the eroded image. The argument SE is a structuring element object or array of structuring element objects returned by the strel function or user defined nxn array. If image is logical and the structuring element is flat, imerode() performs binary erosion; otherwise it performs grayscale erosion. Here SE is an array of structuring element objects; imerode() performs multiple erosions of the input image, using each structuring element in SE in succession. The binary erosion of $A$ by $B$, denoted $A$ $\ominus B$, is defined as the set operation $A \ominus B=\left\{z \mid\left(B_{z} \subseteq A\right\}\right.$. In other words, it is the set of pixel locations $z$, where the structuring element translated to location $z$ overlaps only with foreground pixels in $A$.

In the general form of gray-scale erosion, the structuring element has a height. The gray-scale erosion of $A(x, y)$ by $B(x, y)$ is defined as:

$(A \ominus B)(x, y)=\min \left\{A\left(x+x^{\prime}, y+y^{\prime}\right)-B\left(x^{\prime}, y^{\prime}\right) \mid\left(x^{\prime}, y^{\prime}\right) \in D^{B}\right\}$, where $D_{B}$ is the domain of the structuring element $B$ and $A(x, y)$ is assumed to be $+\infty$ outside the domain of the image.

Most commonly, gray-scale erosion is performed with a flat structuring element $(B(x, y)=0)$. Grayscale erosion using such a structuring element is equivalent to a local-minimum operator:

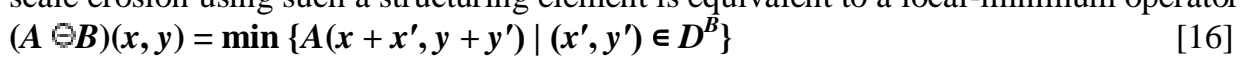

Apply convolute operation to smooth the mask. In order to decide which pixels belong to the shadow, we engage two approaches [2]:

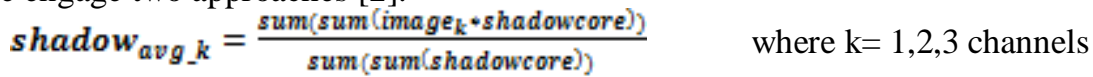

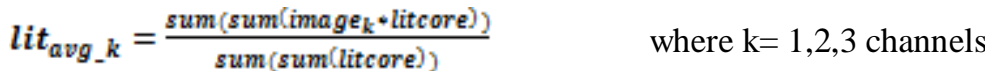

\section{We first calculate average pixel intensities for 3 channels.}

a) We consider being part of the shadow those pixels that have the intensity lower than $60 \%$ of the full average.

b) Compute the non-shadow point's average for the sliding window. We consider being part of the shadow, the pixels that have the intensity lower than the $70 \%$ of the window's average.

This phase finds the lower intensities areas in the picture, and this can be darker object or darker textures not related to shadows (false detection). The result of the shadow detection is a binary shadow mask, which will be the input to the shadow removal phase. Shadow removal phase starts with computing ratio of the luminance of the directed and global lights.

$$
\text { Ratio }_{k}=\text { lit }_{\text {avg_k }_{-}} / \text {shadow }_{\text {avg }{ }_{k}}-1 \quad \text { where } \mathrm{k}=1,2,3 \text { channels [3] }
$$

i.e., For RGB color space it can be expanded as follows:

$$
\begin{aligned}
& \text { Ratio }_{\text {red }}=\text { lit }_{\text {avg_red }} / \text { shadow }_{\text {avg_red }}-1 \\
& \text { Ratio }_{\text {green }}=\text { lit }_{\text {avg_green }} / \text { shadow }_{\text {avg_green }}-1 \\
& \text { Ratio }_{\text {blue }}=\text { lit }_{\text {avg_blue }} / \text { shadow }_{\text {avg_blue }}-1
\end{aligned}
$$


We use a simple shadow model, where there are two types of light sources: direct and ambient light. Direct light comes directly from the source, while environment light is from reflections of surrounding surfaces. For shadow areas part or all of the direct light is occluded.

The shadow model can be represented by following formula:

$$
I_{i}=\left(t_{i} \cos \theta_{i} L_{d}+L_{e}\right) R_{i}
$$

$-\mathrm{I}_{\mathrm{i}}$ represents the value for the $i$-th pixel in RGB space

$-\mathrm{L}_{\mathrm{d}}$ and $\mathrm{L}_{\mathrm{e}}$ represent the intensity of the direct light and environment light also measured in RGB space.

$-\mathrm{R}_{\mathrm{i}}$ is the surface reflectance of that pixel

$-\theta_{\mathrm{i}}$ is the angle between the direct lighting direction and the surface norm

$-t_{i}$ is the attenuation factor of the direct light if $t_{i}=1$ means the object point is in a sunshine region, else if $t_{i}=0$ then the object point is in a shadow region

We denote by $\mathrm{k}_{\mathrm{i}}=\mathrm{t}_{\mathrm{i}} \cos \theta_{\mathrm{i}}$ thse shadow coefficient for the $i$-th pixel and by $\mathrm{r}=\frac{L_{d i}}{L_{z}}$ the ratio between direct light and environment light.

\section{Ratio Calculation and Pixel Relighting}

Based on this model, our goal is to relight each pixel using this coefficient in order to obtain a shadow free image. The new pixel value is computed based on the model proposed in [2]:

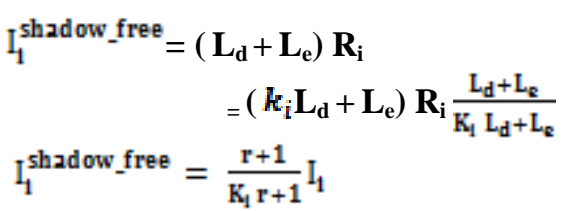

Thus shadow removal formula is

Result $_{\mathrm{k}}=\frac{\text { Ratio }_{\mathrm{k}}+1}{\left(\left(1-\text { smoothmask }^{\prime} \cdot \text { Ratio }_{k}+1\right) \cdot \text { Image }_{k}\right.} \quad$ where $\mathrm{k}=1,2,3$ channels

Resultant image is shadow free image with shadow enhanced pixels. This shadow removal formula mainly depends on mask value and structuring element strel.

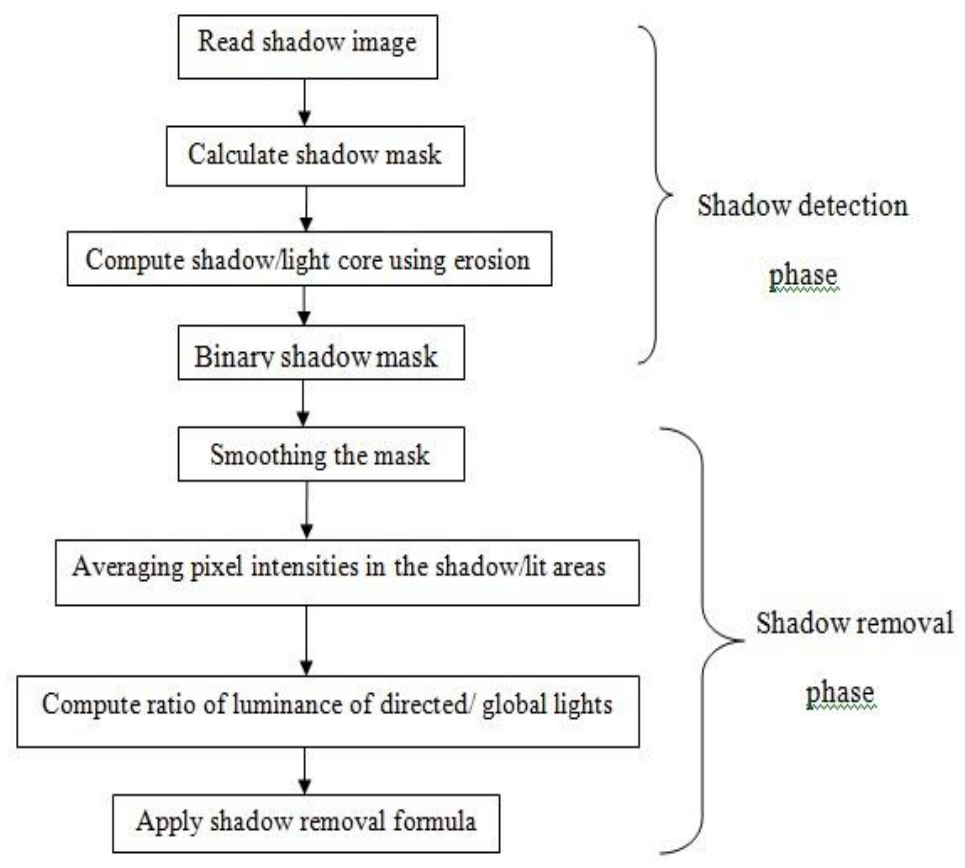

Fig 2. Block diagram of proposed approach.

Experiments on no of images have been done to show the performance of proposed approach. We show results overall and individually for shadow and non-shadow regions (according to the binary ground truth labels). The "non-shadow" regions may contain light shadows, so that error between original and ground truth shadow-free images is not exactly zero for these regions. To show that matting helps achieve smooth boundaries, we also compare the recovery results using only the detected hard mask. We also show results using 
soft matte generated from the ground truth hard mask, which provides a more accurate evaluation of the recovery algorithm.

\section{RESULTS}

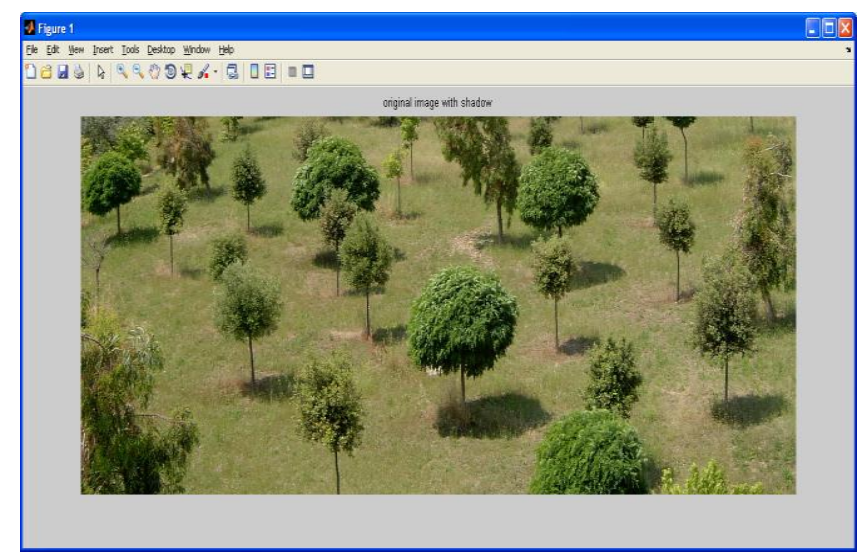

Fig 1: Original Image With shadow

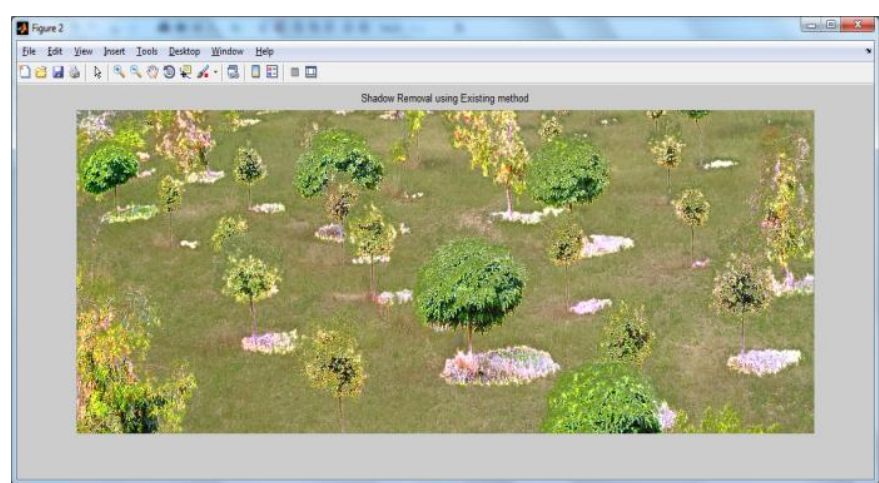

Fig 2: Shadow removal using Existing method considering SE $3 \times 3$ array. It yields more light to shadow areas than surrounding areas returning false erosion.

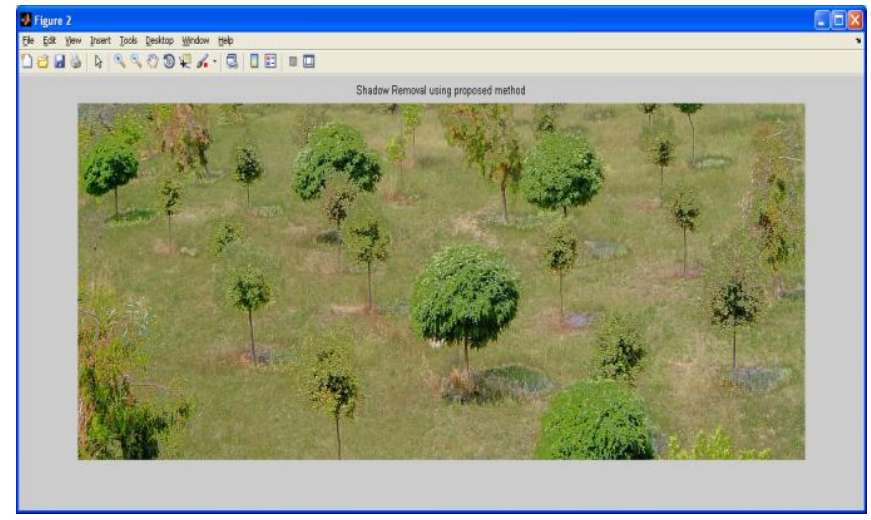

Fig 3: Shadow removal using proposed Method

Here based on SE value pixels under shadow area have same intensities with surrounding values. We consider SE as $5 \times 5$ array.

\section{CONCLUSION}

Shadow removal is main part in image processing applications as it reduces impact of objects. All previous methods follow illuminant invariant image method to remove shadow which is most computationally expensive. This proposed method is easy to implement as it directly uses RGB color space without any Laplacian or gradient transforms of image. Experimental results show that proposed method is appropriate to minimize shadow effect by enhancing luminance of shadow area. This method is easy to implement without errors. In conclusion, we proposed a novel approach to detect and remove shadows from a single still image and complex images. 


\section{REFERENCES}

[1] Li Xu Feihu Qi Renjie Jiang (2006), "Shadow Removal from a Single Image", IEEE Sixth International Conference on Intelligent Systems Design and Applications,. ISDA '06, Vol. 2, pp. 1049-1054.

[2] Ruiqi Guo Qieyun Dai Derek Hoiem (2011), "Single-Image Shadow Detection and Removal using Paired Regions", 2011 IEEE Conference on Computer Vision and Pattern Recognition (CVPR), pp. 2033-2040.

[3] Yael Shor Dani Lischinski (2008), "The Shadow Meets the Mask: Pyramid-Based Shadow Removal", Computer Graphics Forum, Volume 27, Number 2, pp 577-586.

[4] Haijian Ma Qiming Qin Xinyi Shen, (2008), "Shadow Segmentation And Compensation In High Resolution Satellite Images", IEEE International conference on Geoscience and Remote Sensing Symposium, Vol. 2, pp: 1036-1039

[5] G. D. Finlayson, M. S. Drew, and C. Lu. (2009), "Entropy minimization for shadow removal”, IJCV, 85(1):35-57,

[6] B.K.P. Horn (1974), "Determining Lightness from an Image", Computer Graphics and Image Processing, vol, 3. pp.277 299,

[7] D.J. Jobson, Z. Rahman, and G. A. Woodell (1997), "Properties and Performance of the Center Surround Retinex", IEEE Trans. on Image Proc., vol. 6, pp.451-462,

[8] Y. Weiss (2001), "Deriving intrinsic images from image sequences", Proc. Int. Conf. Computer Vision,

[9] Y. Matsushita, K. Nishino, K. Ikeuchi, M. Sakauchi (2004), "Illumination Normalization with Time-Dependent Intrinsic Images for Video Surveillance", IEEE Trans. Pattern Anal. Machine Intell. 26(10):1336-1347.

[10] J.-F. Lalonde, A. A. Efros, and S. G. Narasimhan (2009), "Estimating natural illumination from a single outdoor ima ge".

[11] J.-F. Lalonde, A. A. Efros, and S. G. Narasimhan (2010)," Detecting ground shadows in outdoor consumer photographs".

[12] A. Levin, D. Lischinski, and Y. Weiss (2008), "A closed-form solution to natural image matting"., 30(2):228-242,.

[13] D. R. Martin, C. Fowlkes, and J. Malik (2004), "Learning to detect natural image boundaries using local brightness, color, and texture cues". 26(5):530-549.

[14] B. A. Maxwell, R. M. Friedhoff, and C. A. Smith (2008), "A biilluminant dichromatic reflection model for understanding images", pp: 503-525.

[15] Gallego, J.; Pardàs, M.;(2010), "Enhanced Bayesian foreground segmentation using Brightness and Color Distortion regionbased model for shadow removal," Image Processing (ICIP), 2010 17th IEEE International Conference on , vol., no., pp.34493452, 26-29.

[16] Zhou Liu; Kaiqi Huang; Tieniu Tan,(2012), "Cast Shadow Removal in a Hierarchical Manner Using MRF," Circuits and Systems for Video Technology, IEEE Transactions on, vol.22, no.1, pp.56-66.

[17] Zhaoxiang Zhang; Yuqing Hou; Yunhong Wang; Jie Qin (2011), "A traffic flow detection system combining optical flow and shadow removal," Intelligent Visual Surveillance (IVS), 2011 Third Chinese Conference on , vol., no., pp.45-48, 1-2.

[18] Kryjak, T.; Komorkiewicz, M.; Gorgon, M.;(2011), "Real-time moving object detection for video surveillance system in FPGA," Design and Architectures for Signal and Image Processing (DASIP), 2011 Conference on , vol., no., pp.1-8, 2-4. [19] Jae-Ung Yun; Hyung-Jin Lee; Paul, A.K.; Joong-Hwan Baek; (2007), "Robust Face Detection for Video Summary Using Illumination-Compensation and Morphological Processing," Natural Computation,. ICNC 2007. Third International Conference on , vol.2, no., pp.710-714, 24-27. 